\title{
EMISSIVITY FACTOR OF THERMOPLASTIC MATERIALS For A Robot Bending Process
}

\author{
Steiner Franz*, Bleicher Friedrich \& Klingenbrunner Martin
}
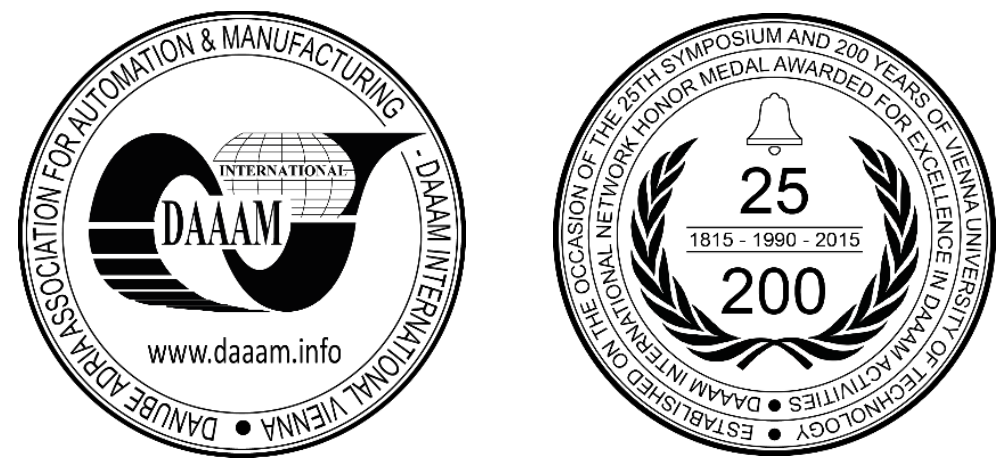

This Publication has to be referred as: Steiner, F[ranz]; Bleicher, F[riedrich] \& Klingenbrunner, M[artin] (2016). Emissivity Factor of Thermoplastic Materials for a Robot Bending Process, Proceedings of the 27th DAAAM International Symposium, pp.0664-0670, B. Katalinic (Ed.), Published by DAAAM International, ISBN 978-3-90273408-2, ISSN 1726-9679, Vienna, Austria

DOI: $10.2507 / 27$ th.daaam.proceedings.096

\begin{abstract}
During thermal incremental forming of semi-finished products, the temperature, spring-back, and morphological structure of the material are interdependent. To determine the forming temperature of the semi-finished product, knowledge about the material-specific properties is required. The processing heat for amorphous polymers is near the glass transition temperature. For semi-crystalline materials, this heating temperature is near the melting point. In approximation to these temperatures, the material can be bent such that a hot core in the semi-finished product leads to low applied force at the robot end effector. Therefore, the semi-finished product is brought near the processing heat by the supply of heat. Subsequently, the core temperature is determined by infrared thermography to observe the heating process. This method provides a connection between the bending force and core temperature, as well as determines the quality of bending.
\end{abstract}

Keywords: infrared thermography; emissivity factor; semi-finished thermoplastic; robot bending

\section{Introduction}

The test field at the Institute of Production Engineering and Laser Technology (IFT) is used for detecting bending parameters with the influence of heat. Bending parameters for forming operations of synthetic materials are the material involved and temperature-dependent spring-back. To investigate material performance, knowledge on the temperature profile during the bending process must be provided. Infrared (IR) thermography, an imaging technique used to display temperatures on object surfaces and for managing production processes, can be used for this purpose. Its main advantage compared with common contact thermometers is contact-free, reactionless temperature measurement, which operates by absorbing thermal radiation from an object. Other advantages include better in-process performance based on computer compatibility and flexibility of thermographic devices. A thermal imaging camera detects and receives this IR radiation. This process is used to characterize materials [1] or measure the bending load of thermoplastic composites as described in [2]. Investigations on damaging impact [3] and linear thermal expansion coefficients of polymeric materials [4] have been reported. The use of IR thermography for controlling a temperature-dependent manufacturing process and rising material temperature and inhomogeneity has been presented [5]. 
Thermoplastic heating methods are used for plasticizing ceramic, quartz, halogen, or carbon radiators [6]. Advantages and disadvantages of different simulation models involving quartz tube heaters or the finite difference method to evaluate material parameters and the heating cycle are demonstrated in [7-8].

\section{Bending robot process}

For the forming of semi-finished plastics, a robot end effector can be used as a bending tool. Further advantages of this arrangement are the possibility of manipulation through the 3D bending space and low bending forces acting on the work piece. The bending line is divided into increments (inc), i.e., the section to be bent is heated, formed, and cooled. Cooling freezes the molecular chains to maintain the bent increment in the desired position in space. The test field at IFT consists of two parts: plastics processing and manipulation via a robot (Figure 1).
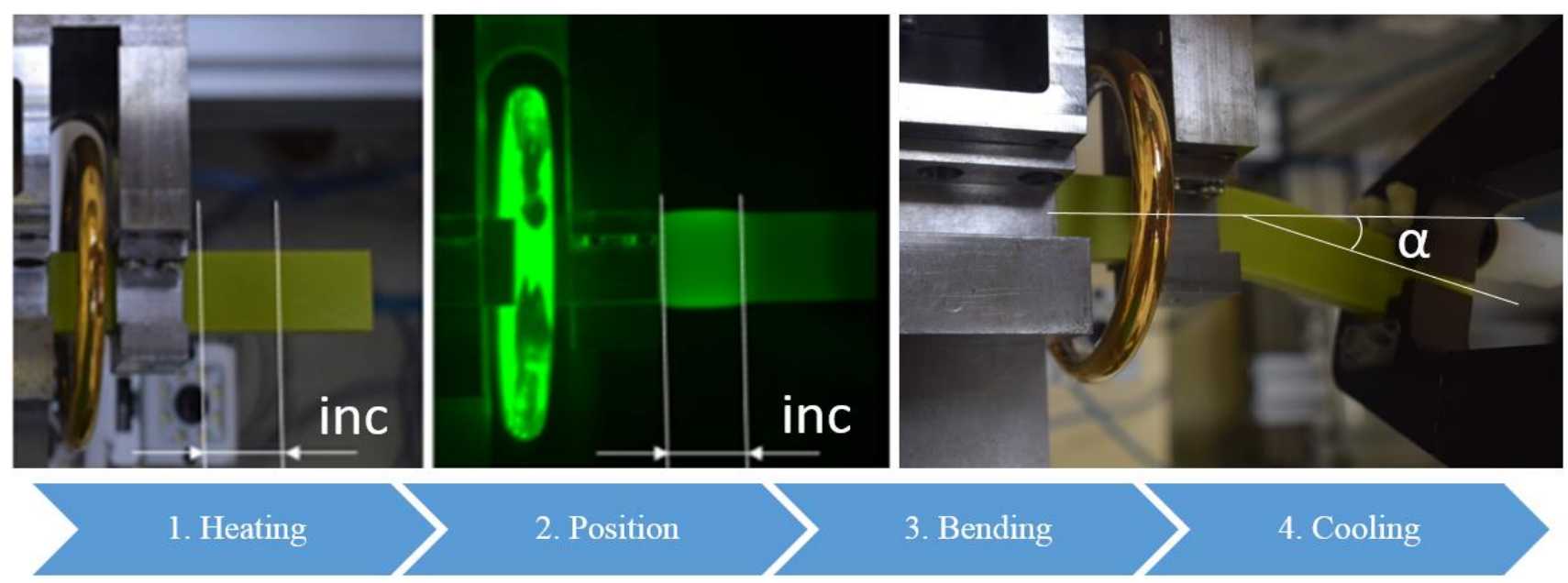

Heating a increment of the semi-finished product (def. performance and duration)

\begin{abstract}
Positioning of the heated increment and fixing of the semi-finished product
\end{abstract}

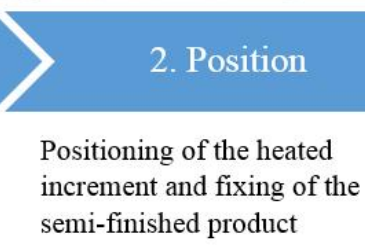

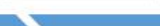
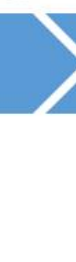

Bending a defined angle ( $\alpha$ for horizontal direction) of the semi-finished product by means of a robot

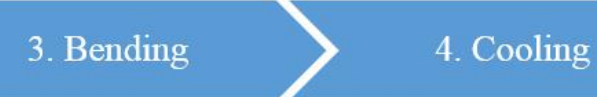

Fig. 1. Bending process: the semi-finished product is heated, positioned, bent (horizontal), and cooled

The process is initiated by the supply of the semi-finished product to the system. The product is then positioned in the heating zone along a linear axis. A circular pronounced IR heating element heats the bent increment until the material reaches the dependent forming temperature field. In this field, the molecular chains are highly flexible and semi-finished products can be plasticized and processed. This plasticized increment is brought into the area outside plastics processing and clamped pneumatically. The area between pneumatic clamping and the end effector is defined as the bending zone. At this position, the end effector engages the material and moves it in space (see Figure 1: $\alpha$ defines the angle of a horizontal bend). The positions in the $\mathrm{x}-, \mathrm{y}-$, and $\mathrm{z}$-directions for movement in space are passed via the control. The bending process data are identified along a polyline and provided as a text file. The content of this text file is converted by machine control into bending parameters. The bending parameters act along the center of gravity of the semi-finished product and define the neutral phase of the bending line. The scanning or design of the CAD file for the front end is described in [9].

For process stability, the temperature curve must be known inside and around the work piece. A test environment is created by an IR camera, which provides information on the external and internal temperatures (core temperature) of the semi-finished product.

Important characteristic properties for the polymer are the glass transition temperature $T_{g}$ and melting temperature $T_{m}$. Below $\mathrm{T}_{\mathrm{g}}$, polymers are very brittle, the structure is "frozen," and polymer chains have limited movement. However, if the temperature increases to $\mathrm{T}_{\mathrm{g}}$, the polymer chains start to vibrate. Above $\mathrm{T}_{\mathrm{g}}$, the material behaves rubbery and the chains can easily move. $\mathrm{T}_{\mathrm{m}}$, an important value for crystalline polymers, varies because of the percentage of crystallinity [10]. Thus, by reaching the forming temperature in the core, low forces for bending are applied to the semi-finished product.

To accurately detect an object's surface temperature, the material and surface-specific emissivity factor $\varepsilon$ must be determined. According to (1), $\varepsilon$ is defined as a factor that compares the specific radiation of a real object ("gray body") to a black body at the same temperature. If the evaluated object is a black body, $\varepsilon$ is equal to 1 . Otherwise, $\varepsilon<1$ (gray body). 


$$
\varepsilon=\frac{M(T)}{\sigma^{*} T^{4}}
$$

Referring to (1), M(T) is the thermal emissivity of the observed object depending on its temperature $\left(\mathrm{W} / \mathrm{m}^{2}\right), \sigma$ is the Stefan-Boltzmann constant, and $\mathrm{T}$ is the temperature of the black body $(\mathrm{K})$, which is equal to the observed temperature. Thermographic devices (e.g., IR cameras) detect $\mathrm{M}(\mathrm{T})$ and calculate the surface temperature with $\varepsilon$ as the input [11].

\section{Determination of the emissivity factors}

To determine the emissivity factors, thermoplastic samples (compare Figure 2 data sheets in [12]) are measured using an IR camera (Optris PI400 properties in [13]) and contact thermometer (Testo 925) for reference purposes. The specimen's surface condition exerts remarkable effects on the emissivity factors. Given the lack of standardized descriptions, the determined emissivity factor must be re-examined every time the supervised specimen's surface varies, even if the material is the same.

The following surfaces supervised by the IR camera can be described as smooth finished after milling them plain.

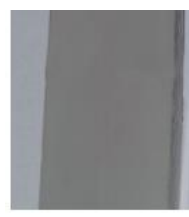

ABS
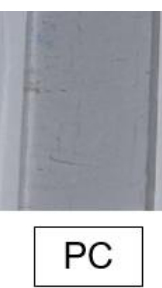
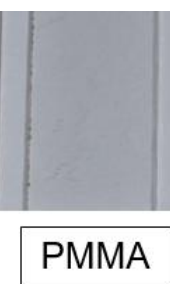
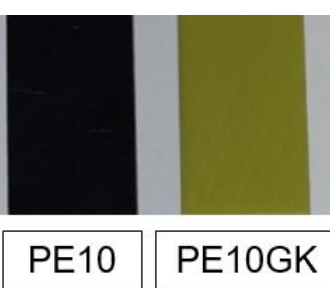
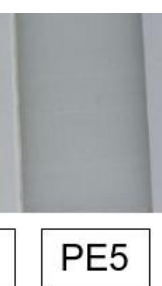
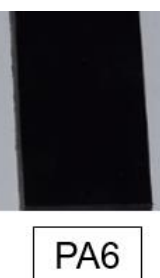

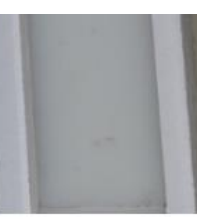

PE3
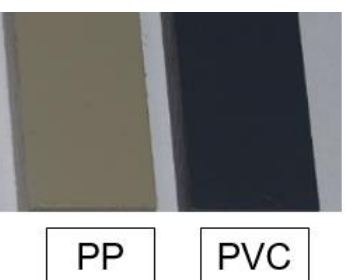

PVC

Fig. 2. Observed samples acrylonitrile-butadiene-styrene (ABS), polycarbonate (PC), poly(methyl methacrylate) (PMMA), ultra-high-molecular-weight (uhmw) polyethylene (PE10), uhmw polyethylene + glassballs, PE10 GB(PE10GK), high-molecular-weight (hmw) polyethylene (PE5), poly(hexano -6-lactam) polyamide 6 (PA6), high-density polyethylene (PE3), polypropylene (PP), and poly(vinyl chloride) (PVC)

First, the samples are prepared (Figure 3$)$. A large hole $\left(d_{1}=3 \mathrm{~mm}, l_{1}>d_{1}\right)$ is needed to realize a black body. According to [11], this size can be achieved by drilling a hole with a depth that is several times its diameter. The second hole is for measuring the core temperature with an ordinary contact thermometer for reference. A small difference between the diameters of the holes and thermometer is essential for precise measurement.

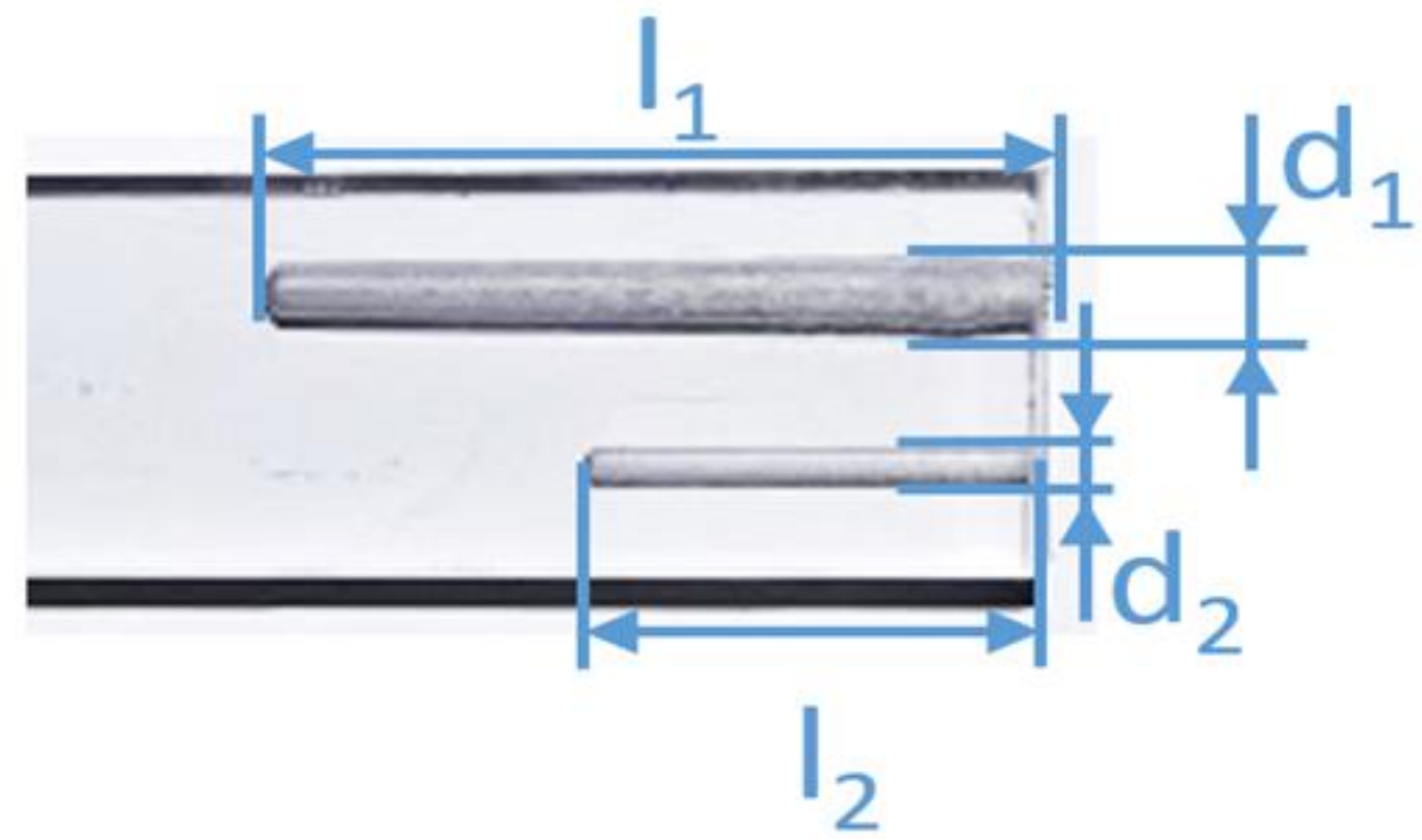

Fig. 3. Preparation of samples of an amorphous semi-finished product poly(methyl methacrylate) 
To reduce external distortion radiation, the whole setup is placed in a black box (Figure 4). The inner surface is coated with a black and rough material to impede reflection. To ensure correct measurement, a distance of at least $500 \mathrm{~mm}$ is necessary between the supervised specimen and IR camera.

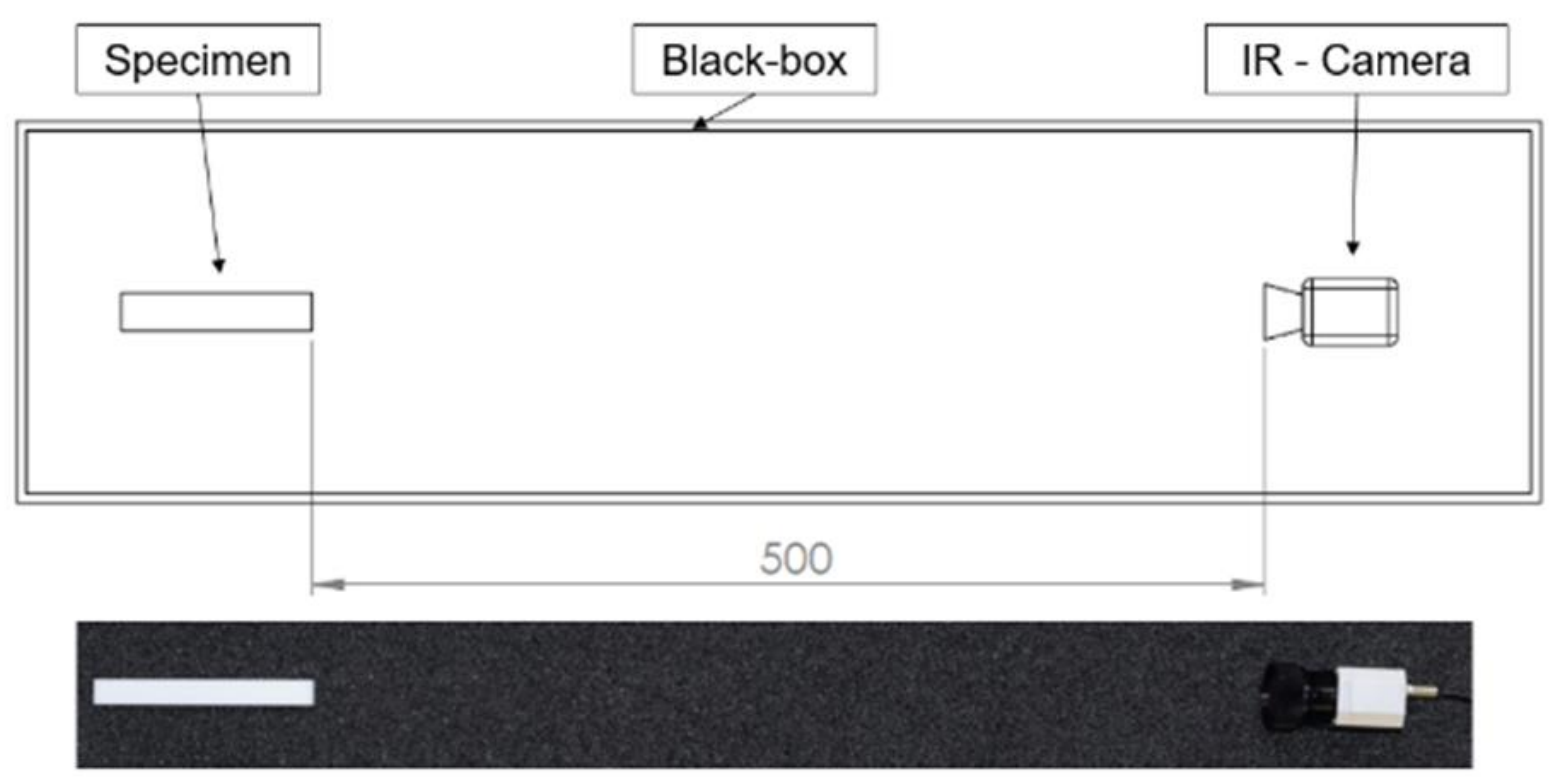

Fig. 4. Experimental setup: top - exemplary, down - real view

Figure 5 shows the surface of one sample, as detected by the IR camera. The two bright spots correspond to the holes in Figure 3. The right cavity shows that the black body has a higher thermal radiation than its surroundings. This feature is due to the ability of a black body to absorb incident thermal radiation completely, with $\varepsilon=1$, because of Kirchhoff's law [11].

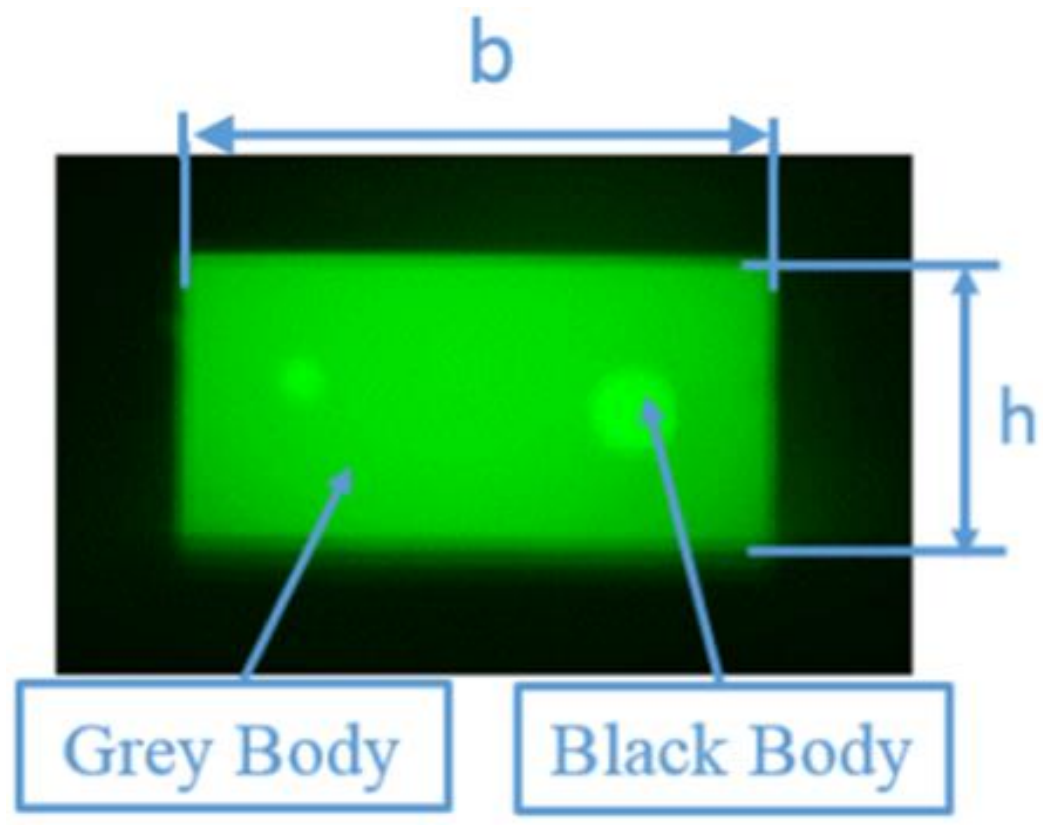

Fig. 5: Surface of the sample through infrared camera

$$
\mathrm{b}=20 \mathrm{~mm}, \mathrm{~h}=10 \mathrm{~mm} \text {. }
$$

Using this knowledge, one measuring point of the processing program evaluates the temperature of the black body with a dedicated $\varepsilon$ of 1 (Figure 6). The second measuring point evaluates the mean surface temperature via the displayed rectangle, excluding the two holes. To achieve the same temperature as the first measuring point (thermal equilibrium) and determine the specific emissivity factor, $\varepsilon$ of the rectangle is modified until the two temperatures are equal [14]. 


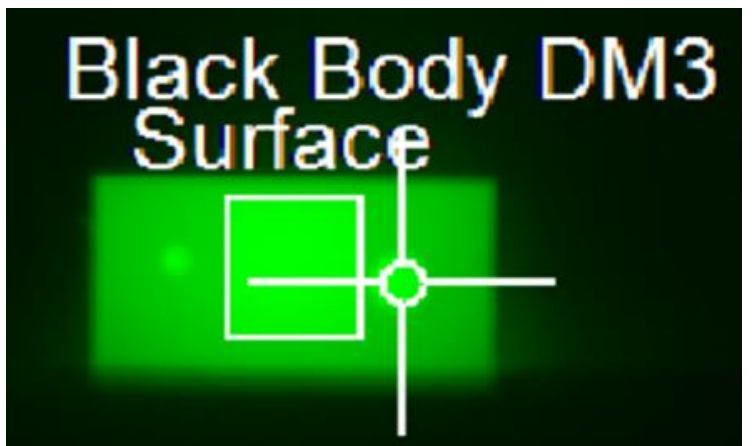

Fig. 6. Measuring surface temperature

\section{Results}

The following Matlab plot (Figure 7) displays the measured temperatures for PE5 and PMMA for amorphous and semi-crystalline materials, respectively. The black and blue lines indicate the black body and surface temperatures over time evaluated by the IR camera, respectively. The red and cyan lines represent the core and surface temperatures measured by an ordinary contact thermometer for reference purposes, respectively. The scale of the vertical axis, as well as the small deviation of the lines, shows suitable results.
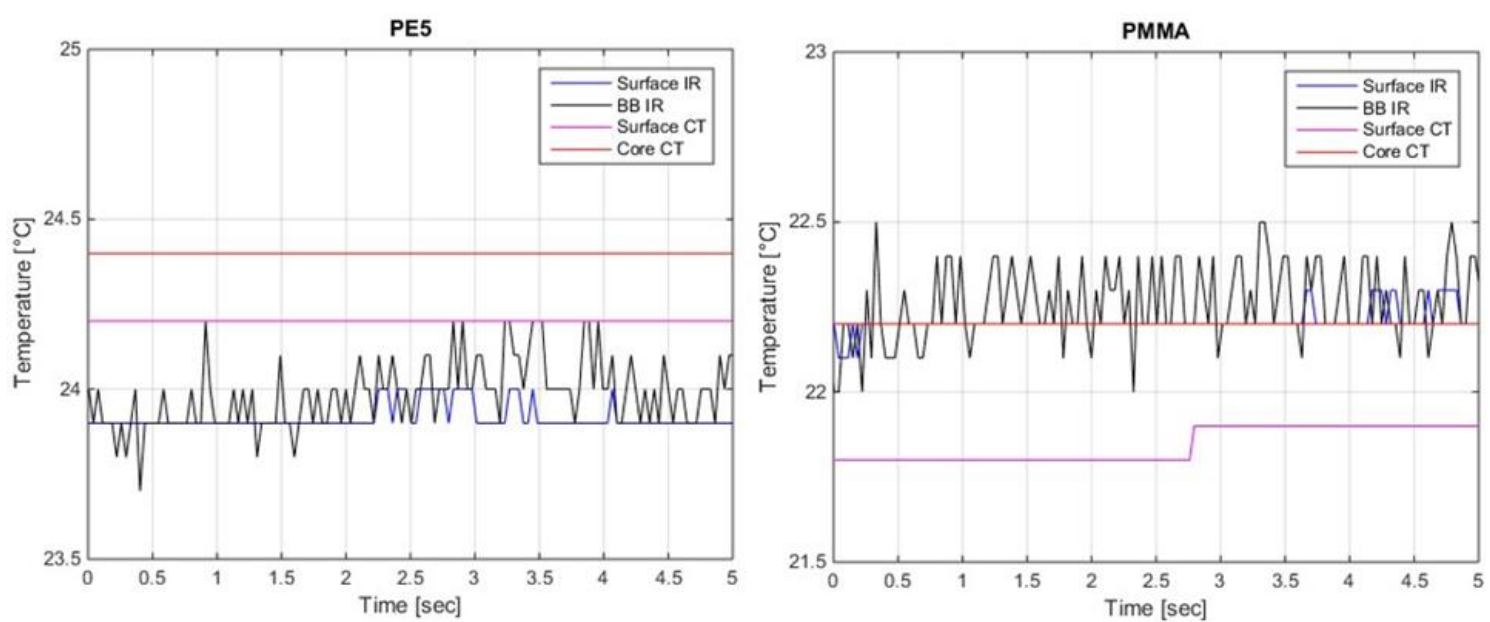

Fig. 7. Visualization of measurement results for PE5 (left) and PMMA (right).

Figure 8 provides an overview of the average measured temperatures over the supervised time on the example polyacrylic (PA6). The first two columns represent the surface and black body temperatures evaluated by the IR camera. The next two columns represent the core and surface reference temperatures measured by the contact thermometer.

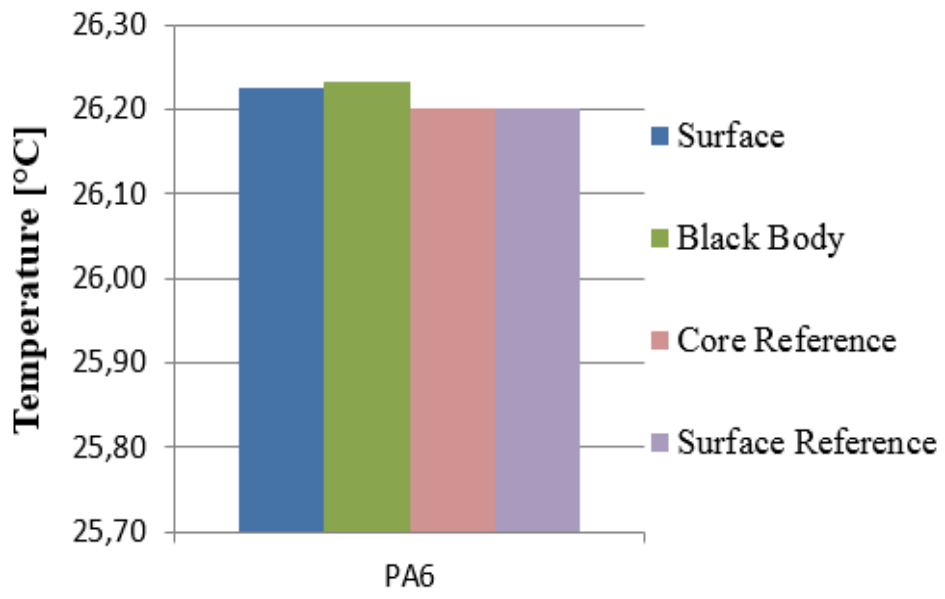

Fig. 8. Measurements of PA6. 
Table 1 shows the determined emissivity factors. Column three describes the relative error (RE), which is evaluated from the mean surface temperature and absolute temperature of the black body (see Figure 8). A small variation of 0.015 in $\varepsilon$ is found across the observed materials.

\begin{tabular}{|c|c|c|}
\hline Sample & $\boldsymbol{\varepsilon}$ & $\begin{array}{c}\text { RE } \\
{[\%]}\end{array}$ \\
\hline ABS & 0,965 & 0,73 \\
\hline PA6 & 0,965 & 0,02 \\
\hline PC & 0,975 & 0,15 \\
\hline PE3 & 0,96 & 0,46 \\
\hline PE5 & 0,965 & 0,02 \\
\hline PE10 & 0,975 & 0,56 \\
\hline PE10GK & 0,965 & 0,03 \\
\hline PMMA & 0,96 & 0,26 \\
\hline PP & 0,965 & 0,28 \\
\hline PVC & 0,965 & 0,25 \\
\hline
\end{tabular}

Table 1. Emissivity factor $\varepsilon$ and relative error of the samples.

The experimental setup provides a simple method to determine the emissivity factor. The effectiveness of this method is displayed in the third column of Table 1 and its small relative error. According to the provided systematic measurement errors of the used devices in Table 2, a measured temperature of approximately $270{ }^{\circ} \mathrm{C}$ is necessary until the RE exceeds the systematic measurement error of the IR camera in the worst case (ABS).

\begin{tabular}{|c|c|}
\hline Systematic measurement error IR & $2{ }^{\circ} \mathrm{C}$ \\
\hline Systematic measurement error CT & $0.5^{\circ} \mathrm{C}$ \\
\hline
\end{tabular}

Table 2. Systematic measurement error of infrared camera and contact thermometer (CT).

\section{Conclusion}

Thermography is a modern and highly useful technology for managing production processes via surface temperature. In addition to the accurate observation of the heating process using the IFT's prototype, $\varepsilon$ may support the selection of materials intended for thermoforming because of Kirchhoff's law. This law states that the absorption of thermal radiation is equal to $\varepsilon$.

The emissivity factor $\varepsilon$ plays a key role in determining radiation, and this relationship can be used as a parameter for process control. Through the detection of contact-free radiation, the temperature can be determined at the surface and in the core. Thus, plasticity in the core will allow application-oriented bending forces in the future.

The emissivity is evaluated by means of a test set-up consisting of different thermoplastic samples and an IR camera embedded in a black box. The lowest value of the tested samples is $\varepsilon=0,965$ (ABS, PA6, PE5, PE10GK, PP, PVC) and the highest value is $\varepsilon=0,975$ (PC, PE10). These values are needed to obtain a statement about the core temperature. This core temperature is crucial for the behavior of the bending line. Higher temperature in the core leads to lower spring-back effects.

The results of this study will benefit future studies, because approximation to glass transition temperature and melting temperature leads to low stress in the material. Stress in the working part during bending affects dimensional tolerance or size accuracy and results in another bending process parameter, the spring-back. The determination of the process parameters and their interrelationships allows reproducible and economic process control.

\section{References}

[1] Meola, C.; Carlomagno, G. M. \& Giorleo, L. (2007) The use of infrared thermography for materials characterization, In: Journal of Materials Processing Technology, Vol. 155-156, (30 November 2004) pp. 1132-1137

[2] Boccardi, S.; Carlomagno, G. M.; Meola, C.; Simeoli, G. \& Russo, P. (2015) Infrared thermography to evaluate thermoplastic composites under bending load, In: Composite Structures, Vol. 134, (15 December 2015) pp. 900904. 
[3] Meola, C.; Boccardi, S.; Boffa, N. D.; Ricci, F.; Simeoli, G.; Russo, P. \& Carlomagnoa, G. M. (2016) New perspectives on impact damaging of thermoset- and thermoplastic-matrix composites from thermographic images, In: Composite Structures, Vol. 152, (15 September 2016) pp. 746-754

[4] Wang, M.; Liao, Y. \& Chen, D. (2013) Determination of linear thermal expansion coefficient of polymeric materials by infrared thermography, In: Polymer Testing, Vol. 32, Issue 2, (April 2013) pp. 175-178

[5] Meola, C.; Giorleo, G.; Nele, L.; Squillace, A. \& Carlomagno, G. M. (2002) Infrared thermography in the quality assurance of manufacturing systems, In: Nondestructive Testing and Evaluation, Vol. 18, Issue 2, (2002) pp. 83-90

[6] Schwarzmann, P. (2015) Heating of Intermediates, In: Kunststoffe 1/2015, Germany: Carl Hanser Verlag, pp. 4851

[7] Brogan, M. T. \& Monaghan, P. F. (1996) Thermal simulation of quartz tube infra-red heaters used in the processing of thermoplastic composites, In: Composites Part A: Applied Science and Manufacturing, Vol. 27, Issue 4, (1996) pp. 301-306.

[8] Fertschej, A.; Langecker, G. R. \& Kertz, J. (2007) Finite Difference Method: Simulation of the Heating Cycle during Thermoforming, In: Kunststoffe 1/2007, Germany: Carl Hanser Verlag, pp. 62-65

[9] Steiner, F. \& Bleicher, F. (2016) Freiformen im sphärischen Biegekopf, In: Kunststoffe 1/2016, Germany: Carl Hanser Verlag, pp. 56-59

[10] Stuart, B. H., Polymer Analysis. ISBN: 978-0-471-81363-7, England: John Wiley \& Sons, Ltd, 2002

[11] Vollmer, M. \& Möllmann, K. P., Infrared Thermal Imaging: Fundamentals, Research and Applications, ISBN: $978-$ 3-527-40717-0, Germany: Wiley-VCH Verlag GmbH \& Co. KGaA, 2010

[12] Faigle Industrieplast $\mathrm{GmbH}$, faigle semi-finished products - plastic technical data, 2009

[13] Optris GmbH, Basic Principles of Non-Contact Temperature Measurement, Germany

[14] Raytek Corporation, The Principles of Noncontact Temperature, USA, 2016 